\title{
Clinicopathological report of children and young adults with nephrotic syndrome undergoing renal biopsy at workshops in Port Harcourt, Nigeria
}

\author{
Christopher Chinedu Obiorah', Philemon Ekemenye Okoro² \\ 'Department of Anatomical Pathology and '2Department of Surgery, University of Port Harcourt Teaching Hospital, Port Harcourt, Nigeria
}

\section{ABSTRACT}

Background: Kidney biopsy is a procedure that is not commonly performed in Nigeria. This study reports the clinical and pathological findings in children who underwent renal biopsies during nephrology workshops held in Port Harcourt in 2014 and 2015.

Methods: The native kidney biopsies were processed and evaluated using light microscopy only.

Results: Nineteen patients ( 10 females) were biopsied. The mean age was $11.0 \pm 6.5$ years and the range was 1.8 to 21 years. Eleven patients had steroid-dependent nephrotic syndrome (SDNS) whereas eight were steroidresistant (SRNS). Hypertension and haematuria were present more often in SDNS. There were II cases (58\%) with non-proliferative lesions, including minimal change disease (4 cases), focal segmental glomerulosclerosis (4 cases) and collapsing glomerulopathy (3 cases). The proliferative lesions ( 8 cases) included membranoproliferative (mesangiocapillary) glomerulonephritis (MPGN, 5 cases), mesangial proliferative glomerulonephritis (2 cases) and MPGN with collapsing glomerulopathy (I case). Overall, the most common pathological diagnosis was MPGN (26\%). Hypertension was more common with proliferative than with non-proliferative lesions (63\% versus 36\%). Conclusions: Although MPGN was the most common morphological lesion among children with nephrotic syndrome, bigger studies are necessary to confirm this. Efforts should be intensified in acquiring the expertise and infrastructure for performing and interpreting renal biopsies in Port Harcourt in order to optimize patient management.

Keywords: renal biopsy; children; Port Harcourt.

\section{INTRODUCTION}

Childhood renal diseases are heterogeneous and vary across geographic regions, depending on genetics, environmental influences and disease awareness $[1,2,3]$. Histological examination of renal biopsy samples is often able to establish the diagnosis and prognosis of renal disease, and guides treatment [4,5]. Although renal biopsy is considered safe in children, it is uncommonly performed in Nigeria, partly because nephrotic syndrome in children is caused mostly by minimal change disease (MCD) and treated empirically with steroid therapy $[6,7]$. Moreover, biopsies in children require more expertise, there is a dearth of nephropathologists in Nigeria, and the need for out-of-pocket payments by patients discourages many from undergoing the procedure [8].

Given the high morbidity and mortality from renal diseases in Nigeria, there is need to study the underlying pathology [9]. Data on the pathology of kidney diseases in children remain scanty. This study reports on the clinico-pathological findings in children with nephrotic syndrome who underwent renal biopsies during workshops held at our centre in Port Harcourt, Nigeria. 


\section{METHODS}

This descriptive study is a review of renal biopsies processed at two interventional nephrology workshops held at the University of Port Harcourt Teaching Hospital (UPTH) in Port Harcourt, in 2014 and 2015. The need to expose nephrologists, residents and pathologists to the performance and pathological interpretation of renal biopsies informed the workshops, which were partly sponsored by the International Society of Nephrology (ISN) and the International Paediatric Nephrology Association (IPNA). Patients were recruited from within and outside UPTH. Ethics committee approval was granted and informed consent was obtained in all cases.

Demographic and clinical information was recorded. This included details on blood pressure, urinalysis, renal function, and serology for hepatitis B, C, and human immunodeficiency virus (HIV).

The biopsies were performed by Malcolm Lewis (Temple Street Children's University Hospital, Dublin, Ireland) using light sedation and spring-loaded semiautomatic biopsy needles with ultrasound guidance. The patients were managed as day cases and discharged home after 5-8 hours of uneventful post-biopsy monitoring. The biopsies were fixed in 10\% neutral buffered formalin and processed in the UPTH laboratory using standard procedures. Due to the lack of immunofluorescence and electron microscopy facilities, only light microscopy was used in evaluating the specimens. Serial sections were stained with haematoxylin and eosin, periodic acid-Schiff stain, Jones methanamine silver and Masson's trichrome. Positive tissue controls were

\begin{tabular}{|lcccc|}
\hline \multicolumn{5}{|c|}{ Table I. Distribution by diagnosis and sex. } \\
\hline Diagnosis & F & M & Total & $\%$ \\
\hline CG & 2 & 1 & 3 & 16 \\
FSGS & 1 & 3 & 4 & 21 \\
MCD & 1 & 3 & 4 & 21 \\
MPGN & 4 & 1 & 5 & 26 \\
MPGN + CG & 1 & 0 & 1 & 5 \\
MSP & 1 & 1 & 2 & 11 \\
Total & 10 & 9 & 19 & 100 \\
\hline
\end{tabular}

CG, collapsing glomerulopathy; FSGS, focal segmental glomerulosclerosis; MCD, minimal change disease; MPGN, membranoproliferative (mesangiocapillary) glomerulonephritis; MSP, mesangial proliferative glomerulonephritis;

F, female; $M$, male.

used for the special stains. In 2014, the slides were prepared by Colleen Jackson and read by Komala Pillay (Red Cross Children's Hospital, Cape Town, South Africa). In 2015, the slides were prepared by Martha Akpanumoh and Berimienyeofori Abbey, and read by Chris Obiorah (UPTH).

\section{RESULTS}

Nineteen patients ( 10 in 2014 and 9 in 2015) underwent renal biopsy. The mean illness duration prior to presentation to the clinic was 22.2 months (range I-132 months) (Table I). Ten patients (53\%) were female. The mean age was $11.0 \pm 6.5$ years (range $1.8-21$ years). Figure । illustrates the distribution of patients by age and sex.

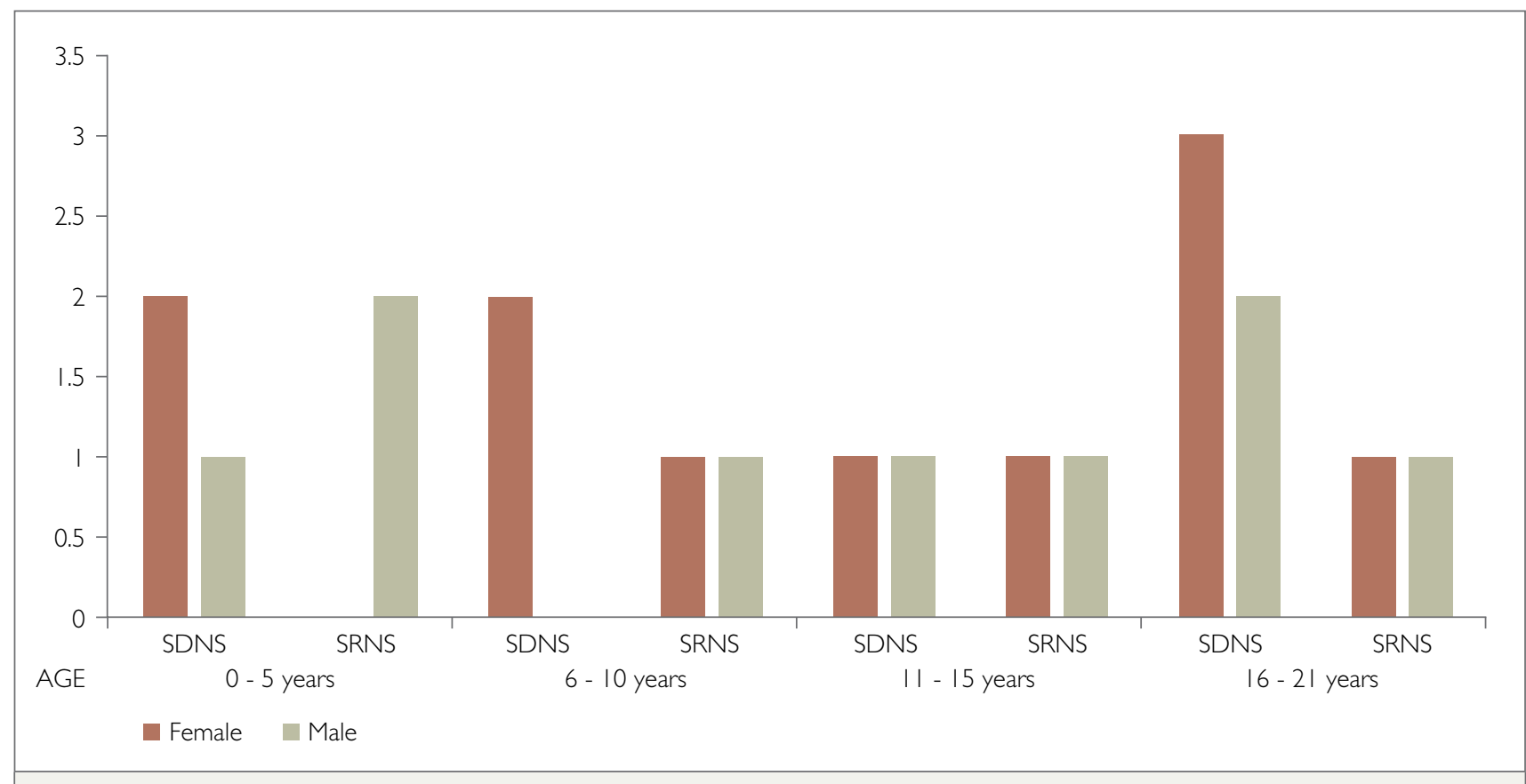

Figure I. Distribution of patients by age, sex and steroid responsiveness. 


\begin{tabular}{|c|c|c|c|c|c|c|c|c|c|c|c|c|}
\hline Case & Sex & Age & Type & Gloms & Sclerosed & Duration & Oedema & HTN & Prot. & Haem. & $\begin{array}{c}\text { Tub } \\
\text { atrophy }\end{array}$ & Diagnosis \\
\hline I & $\mathrm{F}$ & 19 & SDNS & 7 & 0 & 26 & I & I & 1 & I & - & MP \\
\hline 2 & M & 17 & SDNS & 10 & 0 & I & I & I & 0 & I & - & MSP \\
\hline 3 & $\mathrm{~F}$ & 1.8 & SDNS & 9 & 0 & 8 & I & 0 & 0 & 0 & - & MCD \\
\hline 4 & $\mathrm{~F}$ & 5.9 & SDNS & 12 & 0 & 20 & I & 0 & 0 & I & 5 & MP \\
\hline 5 & M & 4 & SRNS & 25 & 0 & I & I & 0 & I & 0 & - & MCD \\
\hline 6 & M & 17 & SRNS & 9 & 4 & - & I & 0 & 1 & I & 5 & MCD \\
\hline 7 & $M$ & 3.9 & SDNS & 10 & 0 & 8 & I & I & 1 & I & - & FSGS \\
\hline 8 & $\mathrm{~F}$ & 4 & SDNS & 14 & 0 & 12 & I & I & 1 & I & - & MSP \\
\hline 9 & $\mathrm{~F}$ & 21 & SDNS & 24 & 5 & 132 & I & I & I & 0 & 10 & MP \\
\hline 10 & $M$ & 2 & SRNS & 17 & 6 & 5 & I & 0 & I & I & 10 & MP \\
\hline 11 & $\mathrm{~F}$ & 17 & SDNS & 23 & 6 & 60 & I & I & I & 0 & 15 & $M P+C G$ \\
\hline 12 & $\mathrm{~F}$ & 17 & SRNS & 35 & 15 & 42 & I & 0 & 0 & 0 & 10 & FSGS \\
\hline 13 & $M$ & 10 & SRNS & 39 & 0 & 3 & I & I & I & 0 & 5 & FSGS \\
\hline 14 & $\mathrm{~F}$ & 6 & SRNS & 30 & 0 & 2 & I & 0 & I & 0 & 20 & $M P$ \\
\hline 15 & $\mathrm{~F}$ & 7 & SDNS & 22 & 0 & 4 & I & I & I & 0 & - & $C G$ \\
\hline 16 & $M$ & 14 & SRNS & 25 & 0 & 10 & I & I & 1 & 0 & - & $C G$ \\
\hline 17 & $\mathrm{~F}$ & 11 & SRNS & 2 & 0 & 3 & I & 0 & 0 & 0 & - & $C G$ \\
\hline 18 & $M$ & 15 & SDNS & 15 & 2 & 38 & I & 0 & 0 & 0 & - & FSGS \\
\hline 19 & M & 17 & SDNS & 6 & 0 & 24 & I & 0 & I & 0 & - & MCD \\
\hline
\end{tabular}

Age, age in years; SDNS, steroid-dependent nephrotic syndrome; SRNS, steroid-resistant nephrotic syndrome; Gloms, number of glomeruli; Sclerosed, number of sclerosed glomeruli; Duration, duration of illness in weeks; HTN, hypertension; Prot., proteinuria; Haem., haematuria; Tub atrophy, percentage of tubular atrophy; I = yes/present; $0=$ no/absent.

All patients were nephrotic, with II cases (58\%) being steroid-dependent (SDNS) and 8 cases (42\%) steroidresistant (SRNS). Hypertension occurred more with SDNS than SRNS (64\% vs 25\%). Haematuria manifested in $46 \%$ of patients with SDNS and in $25 \%$ of those with SRNS.

All the biopsies contained renal cortex and medulla. There were no biopsy-related complications. On average, 17.5 glomeruli were examined in each case (range 2-39). The patients were grouped by their renal histology into those with proliferative glomerular lesions and those with nonproliferative lesions. There were II patients (58\%) with non-proliferative lesions, including minimal change disease (4 cases), focal segmental glomerulosclerosis (4 cases) and collapsing glomerulopathy (3 cases). The 8 patients (mesangiocapillary) glomerulonephritis (MPGN, 5 cases), mesangial proliferative glomerulonephritis (MSP, 2 cases) and MPGN with collapsing glomerulopathy (I case). Overall, the single most common pathological diagnosis was MPGN (26\%).

Females tended to have more proliferative lesions and males more non-proliferative lesions. Hypertension was more prevalent among patients with proliferative lesions (63\% versus $36 \%, p=0.26$ ). Tubulointerstitial lesions were not prominent in the biopsies studied. They were seen in I $1 \%$ and mostly associated with CG.

\section{DISCUSSION}

Percutaneous renal biopsy is considered safe in children [I], yet it is rarely performed in our centre. An earlier study 
from our centre by Anochie et al. indicated that only 14\% of 28 children with nephrotic syndrome underwent renal biopsy and therefore had pathological diagnoses [10]. A study among children in Ibadan, in south-western Nigeria, yielded only 56 biopsies in eight years. The authors noted that renal biopsy was limited to patients who could afford it [I I]. Okpechi et al. reported a low rate of renal biopsies in their review of renal biopsies across Africa. They attributed this to the system of out-of-pocket payments for medical services and the dearth of skills for renal biopsy performance and histological interpretation. [12]. In contrast, a study from Ile-Ife in Nigeria included 264 biopsies over 10 years whereas in Kano, Nigeria, 36 renal biopsies were reviewed in a year $[13,14]$.

There is poor consensus on the indications for renal biopsies in children [15]. For instance, in Ibadan, there was a shift in the indication based on the trend of prevailing causes of nephrotic syndrome [I I]. Nephrotic syndrome is the commonest indication for renal biopsy in most centres in Nigeria. In our study, SDNS predominated as the main indication. This is consistent with previous studies in our centre, which showed high rates of steroid responsiveness since the steroid-responsive patients may become steroid dependent [I0]. In contrast, similar studies conducted in Ibadan and Kano, Nigeria, and those in India and Greece, showed a preponderance of SRNS over SDNS $[4,13,14,17]$. In France, Finland and Italy, the commonest indication is asymptomatic urinary abnormalities [18].

The higher incidence of hypertension and haematuria in SDNS contrasts with the report from Kano with a higher incidence of haematuria in SRNS (80\%) [14]. This difference is probably related to environmental influences on the different populations.

In their study in New York, Samih et al. noted that 57\% of proliferative glomerulonephritis displayed features of MPGN, comparable with our findings. On further evaluation, they observed underlying conditions such as heavy and light-chain deposition diseases, type I cryoglobulinaemic glomerulonephritis, immunotactoid glomerulonephritis, as well as amyloidosis [19]. Owing to our limited diagnostic infrastructure, we were unable to rule out these diagnoses in our cases. In our environment, proliferative glomerulonephritis has been considered a sequela of infectious processes [20]. The predominance of non-proliferative lesions in this study suggests a lesser emphasis on infectious causes.

FSGS and MCD were common diagnoses in our study. The high incidence of FSGS is consistent with previous reports from our centre and other centres in Nigeria $[10,13,14,16]$. However, previous Nigerian studies reported a low incidence of MCD [2 I, 22]. In Ibadan, Asinobi et al. reported a progressively increasing incidence of FSGS over decades, overtaking quartan malarian nephropathy and MPGN to become the most prevalent pathological lesion responsible for childhood nephrotic syndrome at the time of their study [2I].

Nearly two-thirds of patients with proliferative lesions presented with hypertension, which is similar to the report from Kano [14]. The 22-month mean duration of illness prior to diagnosis is in keeping with the trend of late presentation of patients in our environment, which often has dire consequences. Creating awareness to aid early recognition of kidney disease would be helpful as would providing health insurance so that patients would not have to pay out of their own pockets.

\section{CONCLUSIONS}

Although MPGN was the most common morphological lesion among the children with nephrotic syndrome in our study, bigger studies are necessary to confirm this. Because renal biopsy remains important for diagnosis, prognosis and treatment, efforts should be intensified in acquiring the required expertise and infrastructure for performing and interpreting renal biopsies in order to optimize patient management. This includes training nephrologists and nephropathologists, and providing the infrastructure for immunofluorescence and electron microscopy.

\section{Acknowledgments}

We wish to acknowledge the ISN and IPNA, as well as Malcolm Lewis and Komala Pillay, for their roles in facilitating the workshops in Port Harcourt in 2014 and 2015.

We are also grateful to Felicia Eke and Ifeoma Anochie for their efforts in ensuring that the workshops were held. Appreciation also goes to Martha Akpanumor and Berimienyeofori Abbey for assistance in processing the biopsies.

\section{REFERENCES}

I. Hodson EM, Willis NS, Craig JC. Corticosteroid therapy for nephrotic syndrome in children. Cochrane Database Syst Rev. 2007; 4:CD001533.

2. Brodehl J. The treatment of minimal change nephrotic syndrome: lessons learned from multicentre co-operative studies. Eur J Pediat. 1991; 150(6):380-387.

3. Ali A, Ali MU, Akhtar SZ. Histological pattern of paediatric renal diseases in Northern Pakistan. J Pak Med Ass. 20I I; 6 I (7): 653-658. 
4. Printza N, Bosdou J, Pantzaki A, Badouraki M, Kollios K, Ghogha C, et al. Percutaneous ultrasound-guided renal biopsy in children: a single centre experience. Hippokratia. 20 I ; 15(3):258-26 I.

5. Chadha V, Warady BA. Epidemiology of pediatric chronic kidney disease. Adv Chronic Kidney Dis. 2005; 12(4):343-352.

6. Saca E, Hazza I, El-Imam O, Kawar M. Spectrum of biopsy-proven renal disease in pediatric age group at King Hussein Medical Center. J Roy Med Services. 2007; I 4(I):34-37.

7. Alebiosu CO, Kadiri S. Percutaneous renal biopsy as an outpatient procedure. J Natl Med Assoc. 2004; 96:1215-1218.

8. Ocheke IE, Okolo SN, Bode-Thomas F, Agaba El. Pattern of childhood renal diseases in Jos, Nigeria: a preliminary report. J Med in the Tropics. 20 I0; 12(2):87-90.

9. Chijioke A, Adebiyi AB. Clinicopathologic study of adult nephrotic syndrome in llorin Nigeria. Niger Med Pract. 2003; 43:28-32.

I0. Anochie I, Eke F, Okpere A. Childhood nephrotic syndrome: Change in pattern and response to steroids. J Natl Med Assoc. 2006; 98:1977-1981.

I I. Asinobi AO, Ademola AD, Okolo CA, Yariah JO. Trends in the histopathology of childhood nephrotic syndrome in Ibadan Nigeria: preponderance of idiopathic focal segmental glomerulosclerosis. BMC Nephrology. 2015; 16:213-221.

12. Okpechi IG, Ameh OI, Bello AK, Ronco P, Swanepoel CR, Kengne AP. Epidemiology of histologically proven glomerulonephritis in Africa: a systematic review and meta-analysis. PLoS ONE. II (3): e0I52203.

13. Onwubuya IM, Adelusola KA, Sabageh D, Ezike KN, Olaofe OO. Biopsy-proven renal disease in Ile-lfe, Nigeria: A histopathologic review. Indian J Nephrol. 20 16; 26( I):16-22.

14. Obiagwu PN, Aliyu A, Atanda AT. Nephrotic syndrome among children in Kano: a clinicopathological study. Niger J Clin Pract. 2014; 17:370-374.

15. Dhaun N, Bellamy CO, Cattran DC, Kluth DC. Utility of renal biopsy in the clinical management of renal disease. Kidney Int. 2014; 85: 1039- 1048.

16. Eke FU. Nephrotic syndrome in Port Harcourt - clinical presentation and response to steroids. Niger J Paed. 1990; 17:59-63.

17. Printza N, Bosdou J, Pantzaki A, Badouraki M, Kollios K, Ghogha Ch, et al. Percutaneous ultrasound-guided renal biopsy in children: a single centre experience. Hippokratia. 20 I I; I5(3):258-26I.

I8. Fiorentino M, Bolignano D, Tesar V, Pisano A, Van Biesen W, D'Arigo G, et al. Renal biopsy in 2015 - from epidemiology to evidence-based indications. Am J Nephrol. 2016; 43:1 - 19.

19. Samih HN, Anjali S, Glen SM, Anthony MV, Gerald BA, Michael BS, et al. Proliferative glomerulonephritis with monoclonal $\lg G$ deposits. J Am Soc Nephrol. 2009; 20:2055-2064.

20. Jennette JC, Olson JL, Schwartz MM, Silva FG. Primer on the pathologic classification and diagnosis of renal disease. In: Jennette JC, Olson JL, Schwartz MM, Silva FG, editors. Hepinstall's Pathology of the Kidney. 6th ed. Philadelphia, PA: Lippincott Williams \& Wilkins; 2007.

21. Asinobi AO, Gbadegesin RA, Adeyemo AA, Akang EE, Arowolo FA, Abiola $O A$, et al. Predominance of membranoproliferative glomerulonephritis in childhood nephrotic syndrome in Ibadan, Nigeria. West Afr J Med. 1999; 18:203-206.

22. Adedoyin OT, Gbelee HO, Adeniyi A. Childhood nephrotic syndrome in llorin. Niger J Paediatr. 200 I; 28:68-72. 